\title{
Enhancing metabolic cross-talk
}

\author{
The field of cellular metabolic research is growing but remains somewhat fragmented. Facilitating the exchange \\ of ideas between cell biologists and the wider metabolism community will create synergies and further advance \\ the field.
}

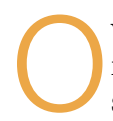
ver the past decade, the field of metabolism research has grown substantially and expanded into many areas of research, such as cancer, immunology, neuroscience, and stem cell and developmental biology, thus affecting biology at all levels, from cells to whole organisms.

Understanding the regulation of cellular metabolism is key for unravelling the principles of cellular homeostasis. An important role in this process involves nutrient sensing and signalling, and the integration of nutritional and metabolic information to regulate cellular energetic and biosynthetic demands. This integration poses a challenge because cellular homeostasis is finely controlled, to the extent that even small deviations from the norm can trigger stress responses, including knock-on effects on proteostasis and the regulation of metabolic processes. Moreover, because cellular metabolic reactions are not restricted to the cytoplasm but are often compartmentalized in organelles, the exchange of metabolic information and metabolites between organelles is crucial for maintaining the activity of cellular metabolic networks and for homeostasis. Although mitochondria have historically been considered the most important metabolic organelles of the cell, other organellesincluding lysosomes, lipid droplets, peroxisomes, endoplasmic reticulum and the Golgi-are now recognized as crucial regulators of cellular metabolism. Furthermore, metabolic communication between the mitochondria and the nucleus highlights an important role of metabolites in gene expression and epigenetics, and therefore in determining cellular decisions and functions. These examples illustrate that cellular metabolism is a complex object of study and requires the integration of knowledge generated by researchers working across many areas of cellular biology.

Because regulation of cellular metabolism is context dependent, the underlying mechanisms vary depending on the nature of the tissue or organ, developmental stage and (patho-)physiological state. As a consequence, cellular metabolic activities are intrinsically linked to tissue function and physiology. The central role of cellular metabolism in regulating cell proliferation, fate and function, for example, is illustrated in the context of cancer, stem cell biology and immunology. Research in these and other fields engaging in the study of cellular metabolism will benefit from increased cross-talk within these disciplines as well as with the wider cell biology community. Moreover, highlighting the similarities and differences among these different biological systems, and using experimental approaches ranging from in vitro to in vivo models will enable a more comprehensive understanding of cellular metabolism and its regulation.

Cellular metabolism is a core subject in the scope of Nature Metabolism, and we strongly believe that enhanced communication among the various scientific communities studying cellular metabolism is important but can be improved. Examples of how we aim to promote such metabolic cross-talk can be found in two recently published Review articles that contrast cellular metabolism in cancer cells, immune and stem cells (Nat. Metab. 1, 16-33; 2019 and Nat. Metab. 1, 177-188; 2019). Another opportunity to increase interaction and collaborations among different fields was provided by the recent Nature Conference in Cellular Metabolism, which Nature Metabolism and Nature organized this month in Xiamen, China (https://www. nature.com/natureconferences/CM19/index. html). This meeting brought together a broad range of researchers studying different aspects of cellular metabolism, including nutrient sensing and metabolic signalling integration; cellular stress responses and proteostasis; organelle metabolism; cancer, stem cell, immunology and vascular biology; and metabolic networks and flux. The aim was to discuss how cells sense their metabolic status and regulate their metabolism, and how such regulation in turn controls cellular signalling and function in different physiological contexts, in both normal and disease settings.

Collaboration and communication across scientific disciplines is fundamental for the progress of science. Nature Metabolism is ideally positioned to offer a platform for discussion that reaches both cell biologists with an interest in metabolism and those studying metabolism in distinct physiological contexts. We believe that fostering cross-talk across the wider metabolism research community will lead to the cross-pollination of ideas and the creation of synergies, with the goal of advancing the understanding of cellular metabolism.

Published online: 12 April 2019 https://doi.org/10.1038/s42255-019-0060-9 